\title{
Benefits of topical fluorides firmly established
}

\author{
Are topical fluoride treatments (TFT; toothpastes, mouthrinses, gels or \\ varnishes) effective in reducing dental caries in children and adolescents?
}

\begin{abstract}
Marinho VCC, Higgins JPT, Logan S, Sheiham A. Topical fluoride (toothpastes, mouthrinses, gels or varnishes) for preventing dental caries in children and adolescents (Cochrane Review). Cochrane Library. 2003; Issue 4. Chichester: John Wiley.

Data sources The Cochrane Oral Health Group's Trials Register the Cochrane Centre Register of Controlled Trials (CENTRAL), MEDLINE, EMbase, SciSearch, Social SciSearch (ISTP Index to Scientific and Technical Proceedings), BIOSIS, CINAHL (Cumulative Index to Nursing and Allied Health Literature), ERIC (Educational Resources Information Centre) define, Dissertation Abstracts and LILACS/BBO (Latin American and Caribbean Health Science Information Database), databases were used, along with searches by hand of relevant journals and the reference lists of included articles. Selected authors and manufacturers were also contacted.

Study selection Randomised or quasi-randomised controlled trials were chosen that had blind outcome assessment and compared fluoride varnish, gel, mouthrinse or toothpaste with placebo or no treatment in children aged up to 16 years over at least 1 year. The main outcome was caries increment measured by the change in decayed, missing and filled tooth surfaces [D(M)FS].
\end{abstract}

Data extraction and synthesis Inclusion decisions, quality assessment and data extraction were duplicated in a random sample of onethird of studies, and consensus was achieved by discussion or a third party. Authors were contacted for missing data. The primary measure of effect was the prevented fraction (PF), that is, the difference in mean caries increments between the treatment and control groups expressed as a percentage of the mean increment in the control group. Randomeffects meta-analyses were performed where data could be pooled. Potential sources of heterogeneity were examined in random-effects metaregression analyses.

Results Of the 144 studies included, 133 contributed data for metaanalysis (involving 65169 children). The D(M)FS pooled PF estimate was $26 \%$ (95\% confidence interval $(\mathrm{Cl}), 24-29 \% ; P<0.0001)$. There was substantial heterogeneity, which was confirmed statistically $(P<0.0001)$, but the direction of effect was consistent.

The effect of topical fluoride varied according to type of control group used, type of TFT, mode/setting of TFT use, initial caries levels and intensity of TFT application, but was not influenced by exposure to water fluoridation or other fluoride sources. The mean $D(M) F S$ PF was on average $14 \%(95 \% \mathrm{Cl}, 5-23 \% ; P=0.002)$ higher in non-placebo controlled trials, and likewise was $14 \%(95 \% \mathrm{Cl}, 2-26 \% ; P=0.25)$ higher in fluoride varnish trials compared with all others. It was $10 \%$ lower $(95 \% \mathrm{Cl},-17$ to $-3 \% ; P=0.003)$, in trials of unsupervised home use compared with self-applied supervised and operator-applied TFT. There was a $0.7 \%$ increase in the PF per unit increase in baseline caries (95\% Cl, 0.2-1.2\%; $P=0.004)$. The numbers needed to treat (NNT) in deciduous and permanent teeth are shown in Table 1 and pooled estimates of the treatment effects of different types of TFT in Table 2.

Address for correspondence: Emma Tavender, Review Group Co-ordinator, Cochrane Oral Health Group, University Dental Hospital of Manchester, Higher Cambridge Street, Manchester M15 6FH, UK. E-mail: emma.tavender@man.ac.uk
Conclusions The benefits of topical fluorides have been firmly established on a sizeable body of evidence from randomised controlled trials. Although the formal examination of sources of heterogeneity

Table 1. NNT in deciduous and permanent dentitions to avoid one new decayed surface.

\begin{tabular}{lcc}
\hline Caries increment/year & $\begin{array}{c}\text { NNT to avoid } \\
1 \mathrm{D}(\mathrm{M}) \mathrm{FS}(95 \% \mathrm{Cl})\end{array}$ & $\begin{array}{c}\text { NNT to avoid } \\
1 \text { defs }(95 \% \mathrm{Cl})\end{array}$ \\
\hline $0.2 \mathrm{D}(\mathrm{M}) \mathrm{FS}$ & $20(18-22)$ & \\
$2.5 \mathrm{D}(\mathrm{M}) \mathrm{FS}$ & $2(2-2)$ & $3.8(2.8-5.7)$ \\
0.8 defs & & $1.6(1.2-2.4)$ \\
1.9 defs & &
\end{tabular}

$\mathrm{D}(\mathrm{M}) \mathrm{FS}$, Decayed missing and filled permanent tooth surfaces; defs, decayed, extracted and filled deciduous tooth surfaces; NNT, numbers needed to treat; $\mathrm{Cl}$, confidence interval.

Table 2. Pooled estimate of treatment effect between different types of TFT from placebo controlled trials.

\begin{tabular}{lcc}
\hline TFT & Preventive fraction & $95 \%$ Confidence interval \\
\hline Varnish & $40 \%$ & $9-72 \%$ \\
Gel & $21 \%$ & $14-28 \%$ \\
Rinse & $26 \%$ & $22-29 \%$ \\
Toothpaste & $24 \%$ & $21-28 \%$ \\
Overall & $26 \%$ & $23-29 \%$ \\
\hline
\end{tabular}

between studies has been important in the overall conclusions reached, these should be interpreted with caution. No definite conclusions about any adverse effects that might result from the use of topical fluorides could be reached because such data from the trials are scarce.

\section{Commentary}

This review adds to a whole series of Cochrane reviews by the same authors dealing with the use of different topical fluoride measures in caries prevention. This time the objectives were to determine the effectiveness and safety of fluoride varnishes, gels, mouthrinses and toothpastes in children and to examine factors potentially modifying their effect.

Only 12 out of the 144 studies included in the review had been published after the 1980s, showing that timely, good-quality literature on the effect of topical fluorides is rare. The review reveals that there is ample evidence on the beneficial effect of topical fluorides in children and adolescents. There was substantial variation in the magnitude of the effect but the direction was remarkably consistent.

The fact that no firm conclusions about possible adverse effects could be reached should not arouse concerns about the safety of topical fluorides. Even if the frequency had been low, noteworthy 
adverse effects attributable to topical fluorides would have been manifested among more than 65000 participants included in the reviewed trials. Lack of observed side effects to be reported is the most likely reason for the paucity of data regarding adverse effects in the literature.

Further confirmation of the well-established benefits of topical fluorides is in no way unexpected. Therefore, the objective to examine factors potentially modifying their effect is much more interesting. There was evidence that the effect of topical fluoride is likely to be greater when used by children who have higher baseline levels of caries experience. There was no evidence, however, that background exposure to fluoridated water or other fluoride sources would have modified the effect of topical fluoride. A higher caries preventive effect was observed with professional applications and supervised self-applications than with unsupervised home use of topical fluorides. An increased total intensity of fluoride application was shown to bring about a higher D(M)FS prevented fraction. Of the different types of topical fluoride measures, the preventive effect was strongest with the use of fluoride varnishes, differences between the other modalities being small. Problems were observed, however, in the methodological quality of the research on the effects of fluoride varnish, although it should be noted that the above findings were based on multiple metaregression analyses these only allow indirect comparisons of the observed effects.

From the clinical point of view, the most important implication of the review is that children and adolescents do benefit from topical fluorides irrespective of possible water fluoridation or other sources of fluoride exposure. Even though there may be differences in the strength of the beneficial effect between different types of topical fluoride, the most critical factors affecting the choice between them are feasibility and costs. At least in Western industrialised countries, home-use of fluoride toothpastes is certainly the most feasible way of exposing the whole population to topical fluoride. Every effort should be made to promote the use of fluoride toothpaste in all groups of children. There is recent evidence that distributing free fluoride toothpaste to children who live in deprived areas can result in a significant reduction in their subsequent caries experience. ${ }^{1}$ For children who have no special risks, regular use of fluoride toothpaste may be sufficient to prevent cavities from occurring. The critical questions are, which children need additional topical treatments, and what type and mode of fluoride use would best suit them. Another Cochrane review by the same group ${ }^{2}$ addresses these questions.

\section{Practice point}

- Children and adolescents benefit from topical fluorides irrespective of water fluoridation or other sources of fluoride exposure.

\section{Hannu Hausen}

Institute of Dentistry, University of Oulu, Oulu, Finland

1. Davies GM, Worthington HV, Ellwood RP, et al. A randomised controlled trial of the effectiveness of providing free fluoride toothpaste from the age of 12 months on reducing caries in 5-6 year old children. Community Dent Health 2002; 19:131-136.

2. Marinho VCC, Higgins JPT, Sheiham A, Logan S. Combinations of Topical Fluoride (Toothpastes, Mouthrinses, Gels, Varnishes) versus Single Topical Fluoride for Preventing Dental Caries in Children and Adolescents (Cochrane Review). Cochrane Library. Chichester: John Wiley; 2004, Issue 1.

Evidence-Based Dentistry (2004) 5, 36-37.

doi:10.1038/sj.ebd. 6400250

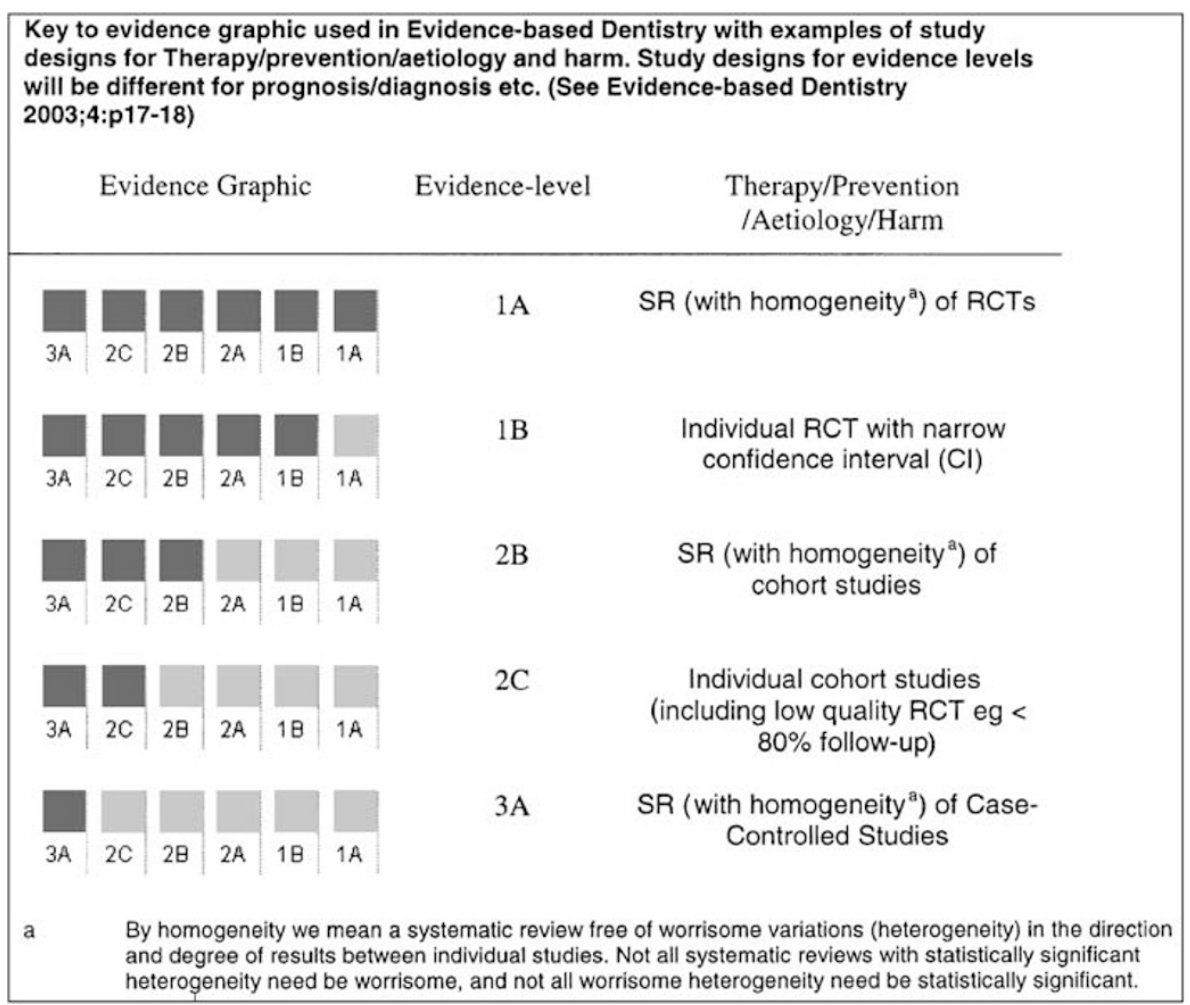

